\title{
Efficient Routing Protocol Algorithm for WIRELESS SENSOR NETWORKS
}

\author{
Sayed Amin Hosseini Seno ${ }^{1}$ \\ ${ }^{1}$ Department of computer science, \\ college of engineering, Ferdowsi \\ University of Mashhad, Iran
}

\author{
Doaa Mohsin Abd Ali ${ }^{2}$ \\ ${ }^{2}$ Department of computer \\ science, college of science, AL- \\ Mustansiriyah University, Iraq
}

\author{
Mohammed Q. Mohammed ${ }^{3,4}$ \\ ${ }^{3}$ Department of Informatics systems management, College \\ of Businesses Informatics, \\ University of Information Technology and \\ Communications, Iraq \\ ${ }^{4}$ Dijlah University College, Iraq
}

\begin{abstract}
Recently, different applications of wireless sensor networks (WSNs) in the industry fields using different data transfer protocols has been developed. As the energy of sensor nodes is limited, prolonging network lifetime in WSNs considered a significant occurrence. To develop network permanence, researchers had considered energy consuming in routing protocols of WSNs by using modified Low Energy Adaptive Clustering Hierarchy. This article presents a developed effective transfer protocols for autonomic WSNs. An efficient routing scheme for wireless sensor network regarded as significant components of electronic devices is proposed. An optimal election probability of a node to be cluster head has being presented. In addition, this article uses a Voronoi diagram, which decomposes the nodes into zone around each node. This diagram used in management architecture for WSNs.
\end{abstract}

Keywords - WSNs, routing protocol, Voronoi, LEACH.

\section{INTRODUCTION}

Recently, there has been a significant focus in designing autonomic WSNs for their utilization in the industry. In previous studies, some protocols, algorithms used for traditional wireless Ad-hoc networks, just like the Ad-hoc on-demand distance vector (AODV), dynamic source routing (DSR). However, the suffusion WSNs applications require more unique features $[1,2]$.

The main approach for designing energy-efficient is clustering, especially providing energyful and highly enhancement with sensor networks [3, 4]. The connections overhead mostly decreased by the regulation clustering, that way gaining decreasing the confusion between the sensor nodes as well as the energy consumption [5-8]. Furthermore, specified node, which is the Cluster Head $(\mathrm{CH})$ main job, is gathering the sensor's data decreasing overall value of data to the main station as well as bandwidth resources and economic energy [912]. The clusters generated by some protocols mostly setup on domestic features which are generally not optimal. Creating load balance energy and effective clusters should giving better importance [13, 14].

In the previous researches, so many research approaches have been used to solve the problem of WSNs. Researchers [15-18] suggest many approaches based on different criteria. Many protocols for WSNs proposed previously assume that the nodes are static. Therefore, new routing algorithms needed to process (topology and mobility) variation in such energy-affected surroundings. Data damaged caused by the mobility of the nodes are possible to disconnect the cluster members from their $\mathrm{CHs}$. In mobility scenario, selecting the $\mathrm{CH}$ by suitable technique to successfully transferring data packets to the main station. To extend the network existence, researchers should consider energy consumption in routing protocols of WSNs.

An enhanced efficient routing protocol for autonomic WSNs was presented in this article. First, the general model for the proposed efficient routing protocol was presented and then an efficient routing scheme for static and mobile WSN was suggested.

In general, the WSNs are consisting quantity of sensor nodes that densely diffused inside or closer to the physical phenomenon, as shown in Figure 1.

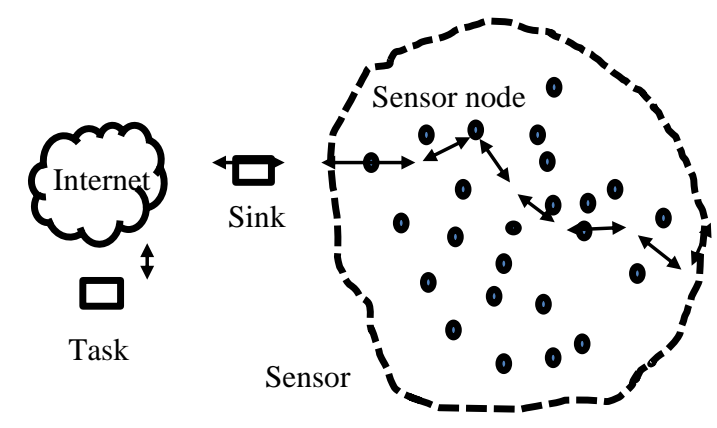

Fig.1: A WSN connected to the internet via sink node [1]

The sensor nodes transceivers usually disperse in the sensor domain where every node capable to gather and transmit data back to the sink/gateway, whiles the end users using a multi-hop infrastructure to minimize the architecture over the sink. Furthermore, by using the sensor nodes processing ability to domestic process illiterate calculation which transfer the required sections processed data only. Through the internet, satellite or any type of wireless network had used to communicate the 
sink with the end users, which made things possible in the internet.

However, in many cases the end-users connected directly from the sink, with possibility to contain multiple sinks and end-users included in the architecture. Some of infrastructure minimal networks like WSNs serve an urgent task in monitoring. Monitoring becomes easier coincidence the improvement of wireless sensing systems. The end-user could get new devices and software progressions are getting available in WSN frequently. The WSNs become complex because the stated fast growth with huge number of devices in the network. Mostly the deployment domain for WSNs is out of the human reach.

The autonomic System, which works independently and rules automatically based on pre-defined rules and gain the recognition via time. The autonomic system has four practical regions for self-management: Firstly, SelfConfiguration represented by automatic arrangement of aggregation. Secondly, Self-healing representing automatic either correction or discovery of errors. Thirdly, Self-Optimization representing automatic monitoring with control of resources to guarantee the optimal working with reverence necessity. Fourthly is the last, Self-Protection representing the protection from arbitrary attacks and proactive identification.

The sensor networks influenced by the low energy obtainable for the sensors based on the lifetime battery. Multi-hop routing will be of more significance for the signal transmission because direct communication it consumes more energy.

To reduce the load in the network, some nodes some have appended duty in hierarchical approach. In location based, the sensor nodes recognition regions exploited to route the request to event from the main station.

The main aim of the proposed article is designing effective routing scheme that perform the incorporate of autonomic computing in designing of the WSN, to build an efficient routing protocol for autonomic WSNs. To gain this aim, this research has focused on the following areas: In order to prolong network lifetime, suggested an improvements with respect to the energy efficiency to the existing cluster-based protocol to static sensor networks. The proposed article, an efficient cluster-based routing protocol for static WSN was explained. In the proposed protocol, the sensor nodes were clustering by a modified FCM to decrease the transferring distance. Moreover, a suitable $\mathrm{CH}$ for every cluster elected based on fuzzy logic system. The parameters (process, energy, concentration and centrality) used for the election. Data that belonging to the clusters send to the Base Station (BS) from the sensors once collected by all the every $\mathrm{CH}$.

Many fuzzy logic algorithms for clustering have been applied in WSNs. In [19], a multi hop clustering proposed, which called fuzzy clustering algorithm, extend the existence of WSNs. This method regulates the $\mathrm{CH}$ radius take into consideration the sensor nodes remaining energy with distance to BS parameters. Researchers in [20] proposed a hierarchical clustering algorithm for $\mathrm{CH}$ selection and cluster formation that used the fuzzy logic method. To determine the $\mathrm{CH}$ selection, three input functions convert the inputs into fuzzy sets, such as distance, nodes density and battery level.

\section{PROTOCOL METHOD}

The chosen protocol represented by centralized protocol, where the process of the clusters which are the formation and head election controlled by BS. The protocol executed in quantity of rounds where in each round passed through the proposed phases, steady state and the setup. The cluster formation, time division multiple access (TDMA) timetable and $\mathrm{CH}$ election decided in the setup stage while the data transmit phase is the steady state phase responsibility. The general model for the proposed routing protocol presented in followed Figure 2.

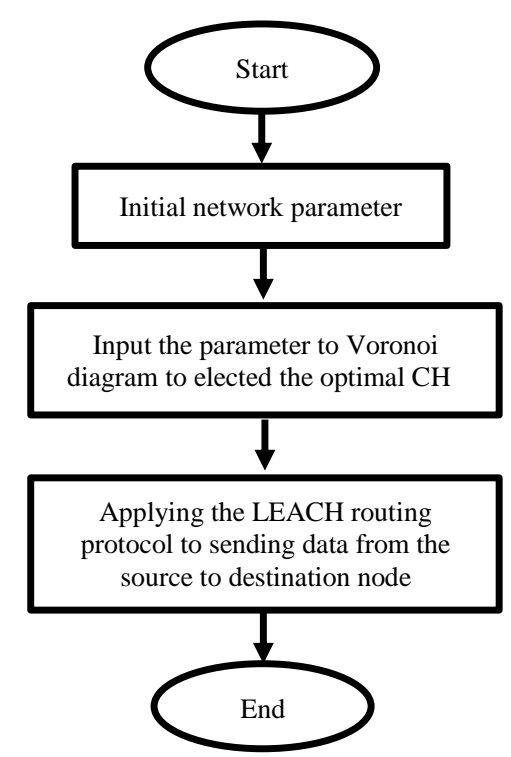

Fig. 2: Protocol structure representation

The detector nodes uniformly clustered for the proposed routing protocol by using a FCM method to reducing the broadcasting distance. Furthermore, the fuzzy logic approach used by some protocol for clustering and backup the head election in mobile WSN.

To select better nodes for clustering the authors in [21] applied the quantity live adjacent nodes and existing power of network nodes. By creating more symmetric clusters, they increased the existence of the sensor network and reduced the power consuming of the sensors. Proposed the Low Energy Adaptive Clustering Hierarchy (LEACH) by [22]. LEACH is a hierarchical protocol in which most nodes transmit to $\mathrm{CH}$ to calculate and compress the data and forward it to the BS. Each round stochastic algorithm used by every node to locate which node would be $\mathrm{CH}$ in the round. LEACH consider every node has a radio powerful appropriate to approach straight the $\mathrm{BS}$ or closest $\mathrm{CH}$, however wasted energy caused if the radio used the full energy interval. Once the CHs turn into nodes, there is not possible to return to be $\mathrm{CHs}$ for $\mathrm{r}$ rounds, where $\mathrm{P}$ represented $\mathrm{CHs}$ required percentage. Afterwards, every node has a $1 / \mathrm{P}$ chance of turning into a $\mathrm{CH}$ in every stage. At every ending stage, the nodes that are not $\mathrm{CHs}$ adopt to connect with the nearest $\mathrm{CH}$. Timetable generated by the $\mathrm{CH}$ for every node in the clusters to transfer the data. Prospect function for candidate turn the node into $\mathrm{CH}$ obtained the beginning value represented by the below equation: 
$T(n)= \begin{cases}\frac{P}{\left(1-P * \bmod \left(r, \operatorname{round} \frac{1}{P}\right)\right)} & \text { if } n \in G \\ 0 & \text { Otherwise }\end{cases}$

Voronoi diagram features have used with the previous equation. By the computation of sensors the sensor scope divided into Voronoi cells, while sensors working directing estimated based on Voronoi vertices [23].

Clustering head selection by using modified LEACH with Voronoi diagram shown in Figure 3 as below.

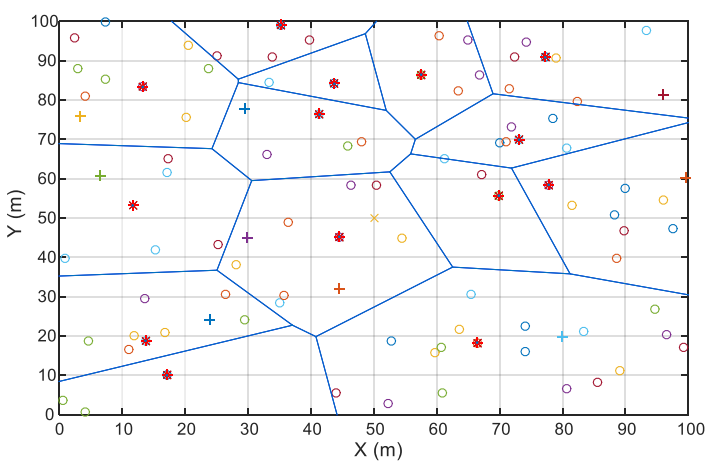

Fig. 3: Clustering head selection with Voronoi cells

In this model, proactive and reactive data reporting is being applied. The proactive networks transmitted the interested data after frequently sensed the perimeter for the nodes therefore, a clear image about the sensed attribute provided at regular intervals. The proactive networks capable for periodic data monitoring to the applications request that. However, in reactive networks, the nodes interacted if unexpected and considerable alteration amount of sensed features. Receiving and transmitting data caused power losing for every sensor node. The power losing calculated based on the range through transmitter and receiver through wireless transmission with free-range broadcast or the multi-path fading models. When the range less than a starting range value $\mathrm{d}_{\mathrm{o}}$ the free-range broadcast model, utilized otherwise multi-path fading channel model utilized.

As indicated in Figure 4, example for radio power losing, the receivers lose power to turn on only the radio electronics while the transmitter lose power to turn on the radio electronics as well as the power amplifier.

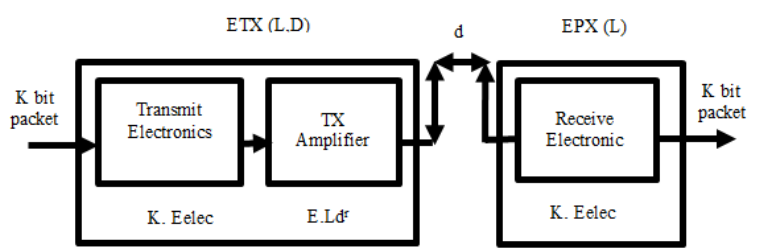

Fig.4: Radio energy dissipation model

Gaining an acceptable indicative to nose ration in transmitting a K-bit through a distance $\mathrm{d}$, furthermore transmitter loss power to broadcast K-bit packet:

$E_{T X}(K, d)=\left\{\begin{array}{c}K E_{\text {elec }}+K \in_{f s} d^{2} \text { ifd }<d_{o} \\ K E_{\text {elec }}+K \in_{m p} d^{4} \text { ifd } \geq d_{o}\end{array}\right.$
Where the threshold $\mathrm{d}_{\mathrm{o}}$ defined by:

$$
\mathrm{d}_{\mathrm{o}}=\sqrt{\frac{\epsilon_{\mathrm{fs}}}{\epsilon_{\mathrm{mp}}}}
$$

Where $\mathrm{E}_{\text {elec }}$ Is the power loss to turn on the electronics loop.

$\epsilon_{f s}$ and $\epsilon_{m p}$ are the transmitter amplifier attributes. In addition, $\mathrm{d}$ represented the range in the midst of two communicating ends.

Energy loss to get a K-bit packet:

$E_{R X}(K)=K E_{\text {elec }}$

Likewise, data gathering in the CHcaused losing energy just like the previous energy growing. EDA represented The Energy Data Aggregation.

\section{THE PROPOSED METHOD}

Two phases of rounds consists in the suggested protocol, which are setup and steady state phases. Every node supposed to become steady; which means the nodes are no longer dynamic when the moment that every node classified. In addition, for simplicity, data packages transferring per round to the BS after produced by each sensor node. The routing protocol proposed model for static WSNs represented in Figure 5. The main goals of setup phase representing by the $\mathrm{CH}$ election and the formation's clusters.

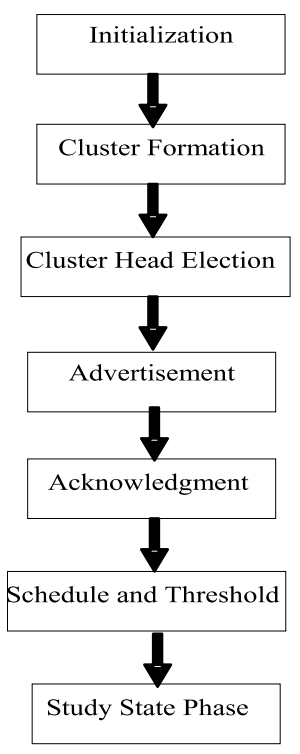

Fig. 5: Proposed Static WSN Protocol Structure.

Initialization 
Starting from setup phase, the base station receiving messages that sensor nodes send. The clusters formation started when the BS received the data.

\section{Cluster Formation}

The sensor nodes clustered utilized by modified LEACH. The primary average of points LEACH algorithm established randomly. The modified algorithm calculates the primary average of points while in the proposed protocol. Reducing the iteration time for creating clusters significantly gain by efficient setting of primary average of points.

\section{Cluster Head Election}

The probability of elected every node itself to be $\mathrm{CH}$ for the existing broadcast to the rest nodes an announcement letter. The non-CH nodes receivers should be on over this phase of setup to fetch the announcement from every $\mathrm{CH}$ nodes. In accordance with this phase is accomplished, every non- $\mathrm{CH}$ nodes determine which cluster they would belong to.

Based on the received signal power of the announcement the decision would be taken. Affecting symmetric spread channels, the $\mathrm{CH}$ announcement comprehended with the maximum signal power is the $\mathrm{CH}$ to whom the minimum value of transmitted power needed for connections. $\mathrm{CH}$ randomly chosen in a similar case of connection.

The $\mathrm{CH}$ node get informed by every node that decided to which cluster it belongs, that nodes would be a member of the cluster. Every node broadcast the date in reverse to the $\mathrm{CH}$. through this stage, all $\mathrm{CH}$ nodes receivers should be on.

Every node that possible be inclusive in the cluster would inform letters to the $\mathrm{CH}$ node. The $\mathrm{CH}$ node creates a TDMA timetable informing every node the time that it would be transmitting based on the quantity of nodes in the cluster while the nodes in the cluster get informed by the schedule transmission.

To reducing the power consuming in nodes, the radio of every non- $\mathrm{CH}$ node should turn off until the node has informed the broadcast time. Otherwise, the $\mathrm{CH}$ node receivers should be on to get the data that sending from the nodes in the cluster.

Base Station computing chances of all nodes then compared with fundamental chances. $\mathrm{CH}$ elected from the cluster the node with the maximum chance. The node with more energy selected to break the relevance among numerous nodes holding the maximum chance. Furthermore, node closest to center is selected. In accordance with the formation of the clusters and $\mathrm{CH}$ election, all sensor nodes know its task from the clusters $\mathrm{BS}$ broadcast the routing information to them such as (CH, non- $\mathrm{CH})$.

\section{Advertisement}

In accordance with selection of $\mathrm{CH}$, keeping Sensor nodes receivers on, so the $\mathrm{CH}$ will broadcast an announcement letter to them.

\section{Acknowledgment}

Sensor nodes after receiving the announcement letter from $\mathrm{CHs}$, furthermore keeping $\mathrm{CHs}$ receivers on to be able to get the acknowledgment letter notified belonging to them from their sensor nodes.

Schedule and Threshold Creation
The acknowledgment received messages from the sensor node, regarding number of nodes the $\mathrm{CHs}$ will create the TDMA schedule to allocate the time for every sensor node in the cluster into cluster members and broadcast it. For the reactivate classification of data broadcast, cluster heads will also broadcast Hard Threshold (HT), which is a value that cluster heads broadcast to all of their members to inform them of its range of interest for the sensed attribute.

The moment that clusters have been generated and the cluster heads are elected, data transmission can begin. The proactive network allowed nodes periodically figure out the environment and transmit the important data. Therefore, they provide a clear image about the figured out attribute at stable periods. Therefore, they are completely suitable for applications demanding frequent data monitoring, while the other classification is appropriate for applications do not require frequent data supervising. The nodes react in reactive network, only to (unexpected, vehement) variation for characteristic behind pre-determined threshold, Hard Threshold (HT); therefore, the sensor node must transfer the figured out data to its $\mathrm{CH}$ if an absolute amount of the characteristic is over this HT.

For monitoring applications, the proactive behavior of WSN has been used for reporting the monitored data in a regular basis. For example, monitor machinery used by wireless sensor network for error discovery and diagnosis. In this type of network, the non- $\mathrm{CH}$, nodes correspondence to their allotted table in each steady-state phase sense the surroundings then transfer sensed data to their $\mathrm{CH}$. The $\mathrm{CH}$ sending aggregated data to the $\mathrm{BS}$. The user should have a clear image of the whole area covered by the network.

While in the wireless sensor network applications that do not require continuous reporting about the sensed attribute, for example (in intrusion detection and explosion detection), the reactive behavior is useful and efficient if the sensed characteristic with range of interest allowing nodes to transmit data to gain reducing the number of transmissions. Consequently, in reactive network, $\mathrm{CH}$ will receive the data from their sensor nodes if sensed value in the domain of interest, above the HT, otherwise, small sized special packet will send by the sensor node notification to $\mathrm{CH}$ referring for is yet alive and checked environment.

\section{SIMULATION RESULTS}

The proposed model simulation result presented by using Matlab software package in this section.

This simulation has focused on prolong network lifetime, suggested an improvements with respect to the energy efficiency to the existing cluster-based protocol to the WSN. The sensor nodes is the proposed protocol, were clustering by a modified LEACH method to decrease the transferring distance. Moreover, a suitable $\mathrm{CH}$ for every cluster elected based on LEACH. The concentration for every node computing by the BS that checking value of every existing node around center node within the area of (100X100) meters. Depending on these, two crisp numbers, when the value of the parameters with the measurement of the membership function crossing point 
will run out the membership function. Each input function linked to the next membership functions which used for representing energy and concentration input parameters, while are used for input parameter representation centrality.

When starting each round the new node architecture created by using LEACH algorithm, optimal election eventuality of a node to become $\mathrm{CH}$ and run the clustering algorithm have been prepared. Taking the root mean square values to calculate the optimum values for number of nodes. In the simulation section, 100 nodes in a $100 * 100$ meter area have been considered. To implement the simulation, several parameters values have to be predefining to run the simulation.

Table 1 represents simulation parameters utilized in the simulation scenarios.

In Figure 6 is being displayed the random distribution of the node sensors. The sensor nodes randomly place by using basic part of code in the given space then aggregate every two distance nodes which is less than or equal to the communication radius.

The connections of each two nodes sensors based on the coverage communication criteria depends on the calculation to find the smallest distance every $\mathrm{CH}$.

\section{TABLE (1): SimULATION PARAMETERS}

\begin{tabular}{|l|l|}
\hline \multicolumn{1}{|c|}{ Parameter } & \multicolumn{1}{c|}{ Value } \\
\hline Number of nodes & 100,200 \\
\hline Network size & $100 * 100$ \\
\hline BS location & $100 * 100$ \\
\hline Data packet size & 4000 bit \\
\hline Initial energy & $0.5 \mathrm{~J}$ \\
\hline Eelec & $50 * 10-9$ \\
\hline Optimal election probability & 0.1 \\
\hline Alpha & 1 \\
\hline Maximum number of rounds & 500 \\
\hline Data aggregation energy & $5 * 10-9$ \\
\hline
\end{tabular}

Using modified LEACH method in the formations of the WSN hierarchy and the $\mathrm{CH}$ selection gaining the randomness of $\mathrm{CH}$ election in $\mathrm{LEACH}$ protocol and uneven distribution of node energy. Simultaneously produce the cluster node consequence through network initialization to reducing the energy consuming when network constantly selects the $\mathrm{CH}$ and prolongs the life cycle of WSN.

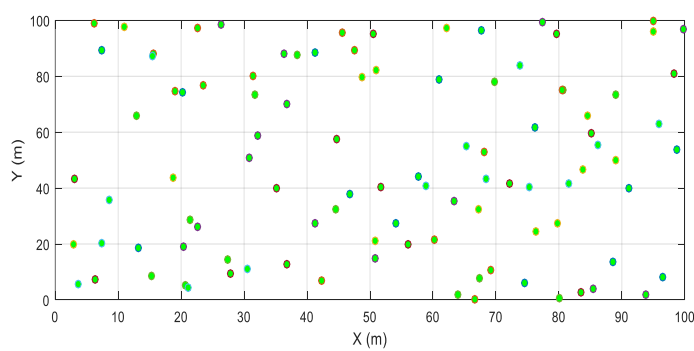

Fig. 6: Distribution of 100 node location sensors.

In Figure 7, Voronoi classification of random distribution of the node sensors. The sensor nodes randomly place by using basic part of code in the given space afterward gathering every group nodes if the distance between every two nodes less than or equal to the communication radius. The proposed design by using a Voronoi diagram to decomposes the range into zone around every node.

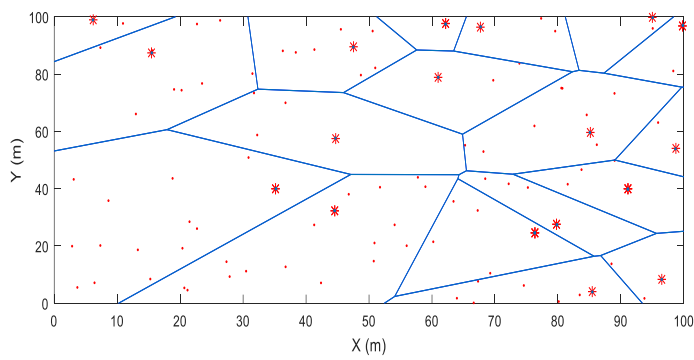

Fig. 7: Voronoi classification of random nodes.

Figure 8 shows the dead nodes value increased with consideration to each round number.

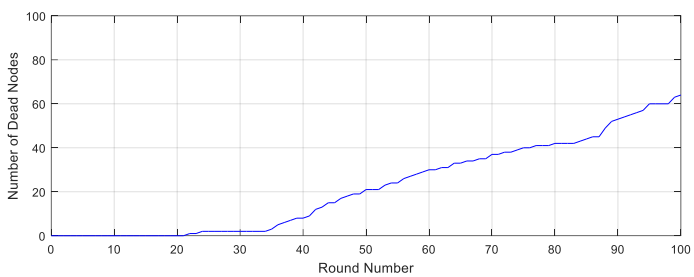

Fig. 8: Number of dead nodes.

Figure 9, shows the decreases behaviors of the corresponding of overall power consuming with consideration to each round number.

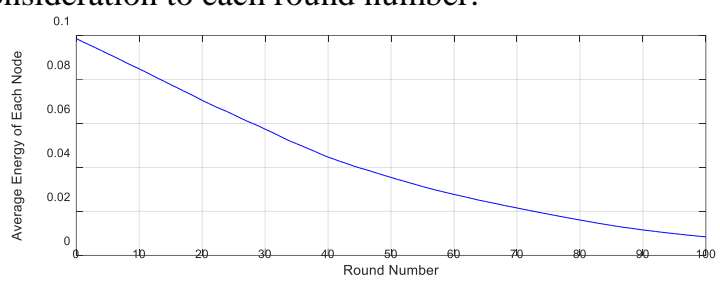

Fig. 9: Corresponding of average energy consumption with respect of number of round.

In Figure 10 is being displayed the random distribution of the node sensors. Randomly distributions of the sensor nodes in specified space between every two nodes connected when the distance less than or equal to the communication radius.

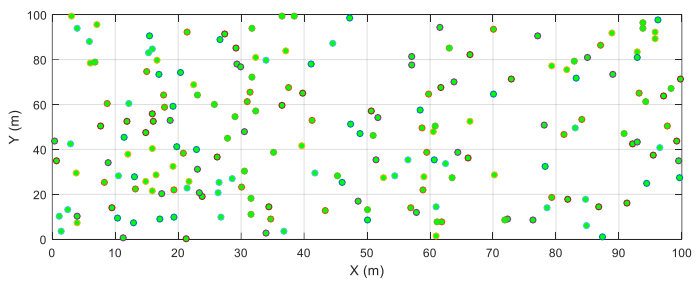

Fig. 10: Random distribution of 200 node sensors.

Figure 11 shows the dead nodes number increase with consideration to each round, the number of nodes is 400 . 


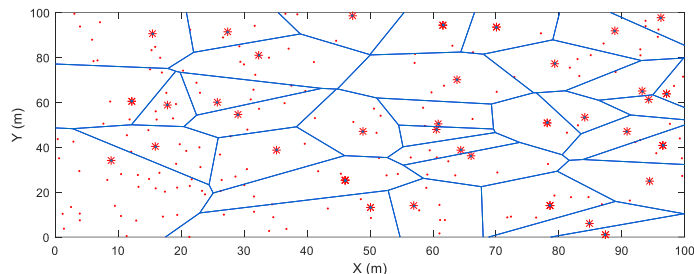

Fig. 11: Voronoi classification of node sensor.

Figure 12 shows the dead nodes number increase with consideration to each round.

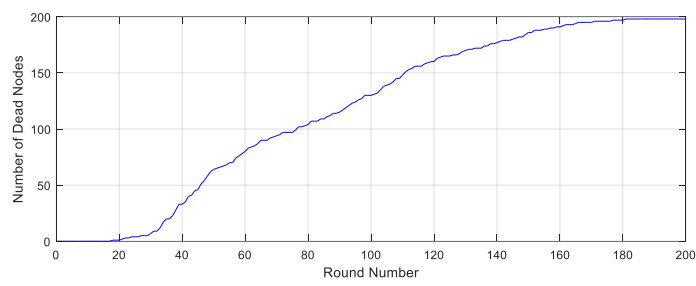

Fig. 12: the dead nodes number increase with consideration to each round.

Figure 13, shows the decreases behaviors of the corresponding of average energy consumption with respect of number of round.

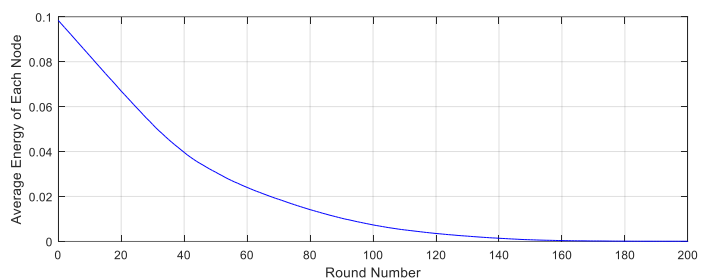

Fig. 13: Corresponding of average energy of each node.

The proposed effectiveness transfer protocols for autonomic WSNs have been evaluated. The time evolution of the corresponding value of packets with consideration to the nodes dead nodes number and the energy consumed as shown in Figure 7.

\section{DISCUSSIONS}

The article goal is to discuss several relevant issues of WSNs, from the application, technology and design. To purpose a WSN, by using the most suitable technology that used and the WSNs protocols to be implemented. Moreover choices based on many factors, as well as some parts taking into consideration when design the WSN. In terms of technologies some possible choices achieved that helping designer in the choice of the most appropriate technology, furthermore potential performance levels are providing. A self-elected $\mathrm{CH}$ collects data position from all sensor nodes in its cluster, calculating and transmitting the decision back to the nodes by using the Voronoi diagram, in a distributed fashion.

Figures (8) and figure (12) give the impression that the dead nodes number increased gradually with consideration to each round.

The modified LEACH performance is compared with a several results found in the researches [21] and [22] as shown in Table (2) below using the same parameters.

TABle (2): Performance OF MOdified LEACH With PREVIOUS RESEARCHES

\begin{tabular}{|l|c|c|c|c|}
\hline \multicolumn{1}{|c|}{ Specifications } & \multicolumn{2}{|c|}{$\begin{array}{c}\text { Modified } \\
\text { LEACH }\end{array}$} & $\begin{array}{c}\text { Fuzzy } \\
\text { Logic[21] }\end{array}$ & $\begin{array}{c}\text { LEACH } \\
{[22]}\end{array}$ \\
\hline Nodes number & 100 & 200 & 100 & 100 \\
\hline Round number & 100 & 200 & 700 & 1400 \\
\hline $\begin{array}{l}\text { Number of } \\
\text { dead nodes }\end{array}$ & $65 \%$ & $99 \%$ & $90 \%$ & $80 \%$ \\
\hline
\end{tabular}

\section{CONCLUSIONS}

Evaluated the effectiveness of the proposed protocol then compared with previous protocols for different kinds of criteria. We compare it against Optimized fuzzy clustering algorithm. This article improved an enhanced efficient routing protocol for autonomic WSN. The general model for the proposed efficient routing protocol and an efficient routing scheme for WSNs. Through simulation results proved the proposed method is found be better sensor nodes. A Voronoi diagram has been applied to solve other problems in a wireless sensor network.

\section{REFERENCES}

[1] Fischione, Carlo. "An introduction to wireless sensor networks." KTH Royal Institute of Technology, Stockholm (2014).

[2] Saleem, K., et al. "A self-optimized multipath routing protocol for wireless sensor networks." International Journal of Recent Trends in Engineering 2.1 (2009): 93-97.

[3] Sivaram, Ponukumati, and Suresh Angadi. "Wireless sensor networks: Routing protocols, challenges, solutions." Interantional Journal of P2P Network Trends and Technology (IJPTT) 3.4 (2013): 214-217.

[4] Asha, ShipraSardana. "Autonomic Wireless Sensor Networks in Real Time Application." International Journal of Computer Science and Communication Engineering IJCSCE Special issue on "Emerging Trends in Engineering" ICETIE 2012.

[5] Mundada, Monica R., et al. "A study on energy efficient routing protocols in wireless sensor networks." International Journal of Distributed and Parallel Systems (IJDPS) Vol. 3 (2012): 311-330.

[6] Ari, Ado Adamou Abba, et al. "A energy efficient clusterbased routing algorithm for wireless sensor networks: Honeybees swarm intelligence based approach." Journal of Network and Computer Applications 69 (2016): 77-97.

[7] Venkateswarlu, K. Muni, A. Kandasamy, and K. Chandrasekaran. "An Energy Efficient Clustering Algorithm for Edge-Based Wireless Sensor Networks."Procedia Computer Science Vol. 89 (2016): 7-16.

[8] Moh'd Alia, Osama. "Dynamic relocation of mobile base station in wireless sensor networks using a cluster-based harmony search algorithm." Information Sciences Vol. 385 (2017): 76-95.

[9] Ouchitachen, Hicham, Abdellatif Hair, and NajlaeIdrissi. "Improved multiobjective weighted clustering algorithm in Wireless Sensor Network." Egyptian Informatics Journal (2016). 
[10] Mann, Palvinder Singh, and Satvir Singh. "Energy efficient clustering protocol based on improved metaheuristic in wireless sensor networks." Journal of Network and Computer Applications Vol. 83 (2017): 40-52.

[11] Zhang, Pengfei, Gaoxi Xiao, and Hwee-Pink Tan. "Clustering algorithms for maximizing the lifetime of wireless sensor networks with energy-harvesting sensors." Computer Networks Vol. 57.14 (2013): Vol. 117 (2017): 6275.

[13] Baranidharan, B., and B. Santhi. "Ducf: Distributed load balancing unequal clustering in wireless sensor networks using fuzzy approach." Applied Soft Computing Vol. 40 (2016): 495-506.

[14] Sabet, Maryam, and HamidrezaNaji. "An energy efficient multi-level routeaware clustering algorithm for wireless sensor networks: Aself-organized approach." Computers \& Electrical Engineering Vol. 56 (2016): 399-417.

[15] Natarajan, Hemavathi, and SudhaSelvaraj. "A fuzzy based predictive cluster head selection scheme for wireless sensor networks." International Conference on Sensing Technology. 2014.

[16] Rana, Sohel, et al. "Fuzzy based Energy efficient multiple cluster head selection routing protocol for wireless sensor networks." International Journal of Computer Network and Information Security Vol. 7.4 (2015): 54.

[17] Srividhya, S., V. Ganapathy, and V. Rajaram. "Fuzzy based Hierachical Unequal Clustering in Wireless Sensor Networks." Indian Journal of Science and Technology Vol. 9.37 (2016).

[18] Shokouhifar, Mohammad, and Ali Jalali. "Optimized sugeno fuzzy clustering algorithm for wireless sensor networks." Engineering Applications of Artificial Intelligence Vol. 60 (2017): 16-25

[19] D. Kumar, T. C. Aseri, and R. Patel, "EEHC: Energy efficient heterogeneous clustered scheme for wireless sensor networks," Computer Communications, vol. 32, pp. $662-$ 667, 2009.

[20] M. A. AbdulAlim, Y. C. Wu, and W. Wang, "A fuzzy based clustering protocol for energy-efficient wireless sensor networks," in Advanced Materials Research, 2013, pp. 685690.

[21] Bidaki, Moazam, and Reza Kamel Tabbakh. "Efficient Fuzzy Logic-Based Clustering Algorithm for Wireless Sensor Networks." International Journal of Grid and Distributed Computing 9.5 (2016): 79-88.

[22] W. R. Heinzelman, A. Chandrakasan, and H. Balakrishnan, "Energy-efficient communication protocol for wireless microsensor networks," in System sciences, 2000. Proceedings of the 33rd annual Hawaii international conference on, 2000 , p. 10 pp. vol. 2.

[23] Sung, Tien-Wen, and Chu-Sing Yang. "Voronoi-based coverage improvement approach for wireless directional sensor networks." Journal of Network and Computer Applications 39 (2014): 202-213. 